\section{(2) OPEN ACCESS}

\title{
Pulmonary vascular proliferation in patients with severe COVID-19: an autopsy study
}

\author{
Belén Pérez-Mies, ${ }^{1,2,3,4}$ María Gómez-Rojo, ${ }^{5}$ Irene Carretero-Barrio 주 , \\ Tommaso Bardi, ${ }^{5}$ Amparo Benito, ${ }^{1,4}$ Mónica García-Cosío, ${ }^{1,4}$ Álvaro Caballero (ㄷ) ${ }^{6}$ \\ Raul de Pablo, 2,4,6 Juan Carlos Galán, 2,7,8 David Pestaña, ${ }^{4,5}$ Jose Palacios (1) 1,2,3,4
}

\begin{abstract}
- Additional material is published online only. To view, please visit the journal online (http://dx.doi.org/10.1136/ thoraxjnl-2020-216714).

'Pathology, Hospital Ramón y Cajal, Madrid, Spain

${ }^{2}$ Instituto Ramon y Cajal de Investigacion Sanitaria, Madrid, Spain

${ }^{3}$ CIBERONC, Madrid, Spain ${ }^{4}$ Universidad de Alcala de Henares, Madrid, Spain ${ }^{5}$ Anesthesiology and Surgical Critical Care Department, Hospital Ramón y Cajal, Madrid, Spain

${ }^{6}$ Medical Intensive Care Unit, Hospital Ramón y Cajal, Madrid, Spain

${ }^{7}$ Clinical Microbiology Unit, Hospital Ramón y Cajal, Madrid, Spain

${ }^{8}$ CIBERESP, Madrid, Spain
\end{abstract}

\section{Correspondence to}

Dr Jose Palacios, Pathology, Hospital Ramón y Cajal, Madrid 28034, Comunidad de Madrid, Spain;

jose.palacios@salud.madrid.org

Received 7 December 2020 Revised 2 February 2021 Accepted 4 March 2021 Published Online First 23 March 2021

\section{Check for updates}

(c) Author(s) (or their employer(s)) 2021. Re-use permitted under CC BY-NC. No commercial re-use. See rights and permissions. Published by BMJ.

To cite: Pérez-Mies $B$, Gómez-Rojo M, CarreteroBarrio I, et al. Thorax 2021;76:1044-1046.

\section{ABSTRACT}

Diffuse alveolar damage and thrombi are the most common lung histopathological lesions reported in patients with severe COVID-19. Although some studies have suggested increased pulmonary angiogenesis, the presence of vascular proliferation in COVID-19 lungs has not been well characterised. Glomeruloid-like microscopic foci and/or coalescent vascular proliferations measuring up to $2 \mathrm{~cm}$ were present in the lung of 14 out of 16 autopsied patients. These lesions expressed CD31, CD34 and vascular endothelial cadherin. Platelet-derived growth factor receptor- $\beta$ immunohistochemistry and dual immunostaining for CD34/smooth muscle actin demonstrated the presence of pericytes. These vascular alterations may contribute to the severe and refractory hypoxaemia that is common in patients with severe COVID-19.

\section{INTRODUCTION}

Patients with COVID-19 pneumonia admitted to the intensive care unit usually present severe, persistent and refractory hypoxaemia. Several vascular mechanisms have been described to explain this severe hypoxaemia, including the loss of hypoxic pulmonary vasoconstriction, vessel enlargement and hyperaemia. Some image studies using CT, functional respiratory imaging on CT and dual-energy CT support the hypothesis of hyperperfusion associated with vasodilation. ${ }^{1-3}$ In addition to vasodilation, vascular proliferation due to local angiogenesis might contribute to increased perfusion. Ackermann et $a l,{ }^{4}$ by using transmission and scanning electron microscopy and microvascular corrosion casts, elegantly demonstrated increased intussusceptive and sprouting angiogenesis in patients with COVID-19. However, at present, the histological manifestations of lung angiogenesis associated with COVID-19 are not well characterised.

In this study, we analyse lung vascularisation by immunohistochemistry in 16 autopsied patients in order to evaluate the role of increased angiogenesis in COVID-19 lung pathology and its potential role in hypoxaemia.

\section{METHODS}

This retrospective series included clinical, pathological and laboratory data from 16 patients with confirmed SARS-CoV-2 infection who were autopsied in our institution. A detailed description of histopathological and immunohistochemical methods is presented in the online supplemental files.
Vascular proliferation was semiquantitatively scored from 1 to 3 on one CD34-stained lung section from each lobe (80 sections in total) (figures 1 and 2). Vascular proliferations consisted of capillary tufting resembling glomeruloid lesions or capillaries arranged perpendicular to each other, forming a complex mesh-like framework (figure 1D-I). The number of such lesions of at least $150 \mu \mathrm{m}$ in size was counted in each CD34stained section. If $\leq 5$ lesions were observed, the section score was 0 . If more than five lesions were observed, it was scored 1. If there was confluence of lesions, occupying at least one low-power field, it was scored 2. Finally, if vascular proliferation measured more than $1 \mathrm{~cm}$ in its maximum diameter, it was scored 3 (figure 2). The final vascular score (VS) from each patient was the sum of individual lobe scores (from 0 to 15 ).

\section{RESULTS}

Clinical, pathological and immunohistochemical data of this series are presented in online supplemental files. Glomeruloid-like and coalescent proliferations expressed CD34, CD31 (online supplemental 3A,B), and vascular endothelial (VE) cadherin (figure $3 \mathrm{~A}$ ), indicating their vascular origin. In addition, single immunostaining with platelet-derived growth factor receptor- $\beta$ and double immunostaining for CD34/SMA (smooth muscle actin) demonstrated the presence of pericytes (figure 3B,C and online supplemental figure 3C,D). Variable Ki67 expression indicated proliferative activity in these lesions (online supplemental figure 4A,B). Dual immunostaining for CD34/ CK18 (cytokeratin 18) differentiated these vascular areas from areas of deficient alveolar expansion or artificial collapse (figures $1 \mathrm{~F}, \mathrm{I}$ and $2 \mathrm{E}$ ).

We found microscopic foci of vascular proliferation in at least one lobe in 14 patients $(87.5 \%)$ (online supplemental figure 6). Eleven patients $(68.7 \%)$ had a VS of 2 or 3 in at least one lobe. The median VS was 5.5 (IQR 3.25). Eight patients (50\%) had a VS equal to or lower than 5 (low VS), and eight patients $(50 \%)$ had a VS of 6 or higher (high VS).

We did not find statistically significant differences between patients with high and low VS in any of the clinical, laboratory or pathological variables analysed (see online supplemental file). A positive significant correlation was observed between VS and age (online supplemental figure 7). 

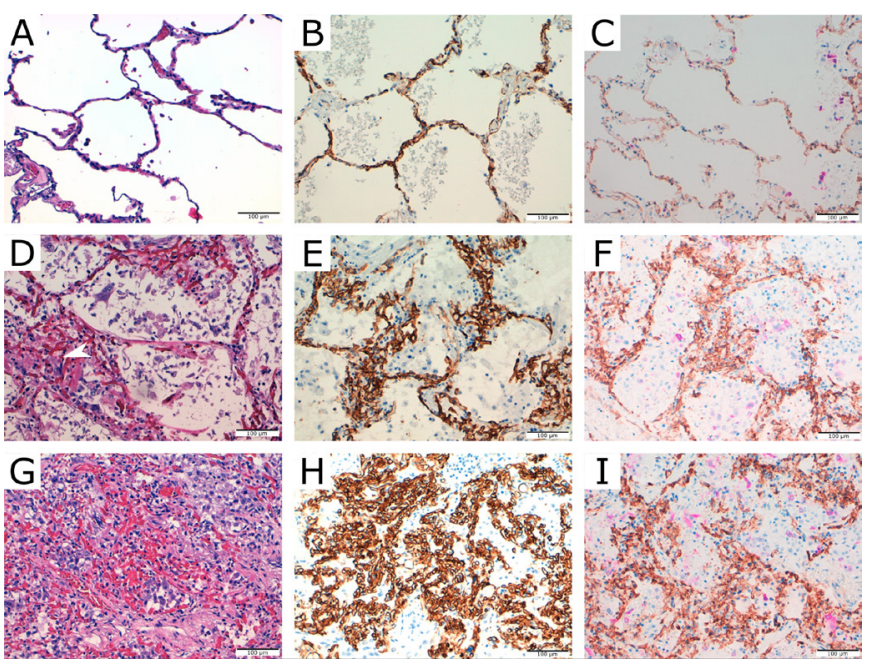

Figure 1 Vascular patterns in patients with COVID-19. (A) Normal lung H\&E. (B) CD34 expression in normal lung. (C) Dual staining for CD34 (brown) and CK18 (pink) in normal lung. (D) 'Glomerularlike' vascular tufting $>150 \mu \mathrm{m}$, protruding into the alveolar lumen or enlarging the alveolar walls (VS 1). (E) CD34 staining. (F) Dual staining for CD34/CK18 with some epithelial cells in alveolar spaces. (G) Confluent capillaries arranged perpendicularly to each other occupying at least one high-power field (VS 2) (H) CD34 staining. (I) Dual staining for CD34/CK18 with scarce residual epithelial cells in alveolar spaces not entrapped in the vascular proliferation. VS, vascular score.

\section{DISCUSSION}

We observed that vascular proliferation is a frequent finding in the lungs of patients with severe COVID-19. Lesions were irregularly distributed across all pulmonary lobes, although they tended to predominate in lower one, and ranged from microscopic glomeruloid-like to coalescent areas greater than $2 \mathrm{~cm}$. In these vascular lesions, endothelial cell proliferation was demonstrated by Ki67 immunostaining. Since we did not observe an association between VS and hospital length of stay and the percentage of diffuse alveolar damage (DAD) lesions, our results suggested that vascular proliferation was not directly related to the process of DAD that was common in these patients.

Despite the relatively high number of COVID-19 autopsy series recently reported, lung vascular proliferation has been only occasionally mentioned. This is probably due to the masking of such changes in the context of severely damaged lungs with DAD lesions. Although capillary congestion has been frequently described, ${ }^{5}$ increased vascularisation has been reported in only two series. In an ultrastructural study without histological correlations, Ackermann et $\mathrm{al}^{4}$ observed increased angiogenesis in five patients. In addition, De Michele et $a l^{6}$ reported frequent microscopic capillary hemangiomatosis ( $\mathrm{PCH}$ )-like lesions, usually detected in patients without DAD histological patterns. In our series, in addition to the microscopic lesions similar to those described by De Michelle et al, we saw confluent lesions, some of them observable at the macromicroscopic level both with the H\&E-stained sections and with sections analysed for CD34, CD31 and VE-cadherin expression. These vascular proliferations differed from those observed in $\mathrm{PCH}$, an infrequent lesion associated with pulmonary hypertension in which the lung architecture is preserved and thickened alveolar septa contain at least two layers of capillaries. ${ }^{7}$ Interestingly, vascular proliferations in the form of $\mathrm{PCH}$ and glomeruloid structures, like those observed in our study, have been described in the lungs of morbid obese patients, a condition that predisposes to severe COVID-19. ${ }^{8}$
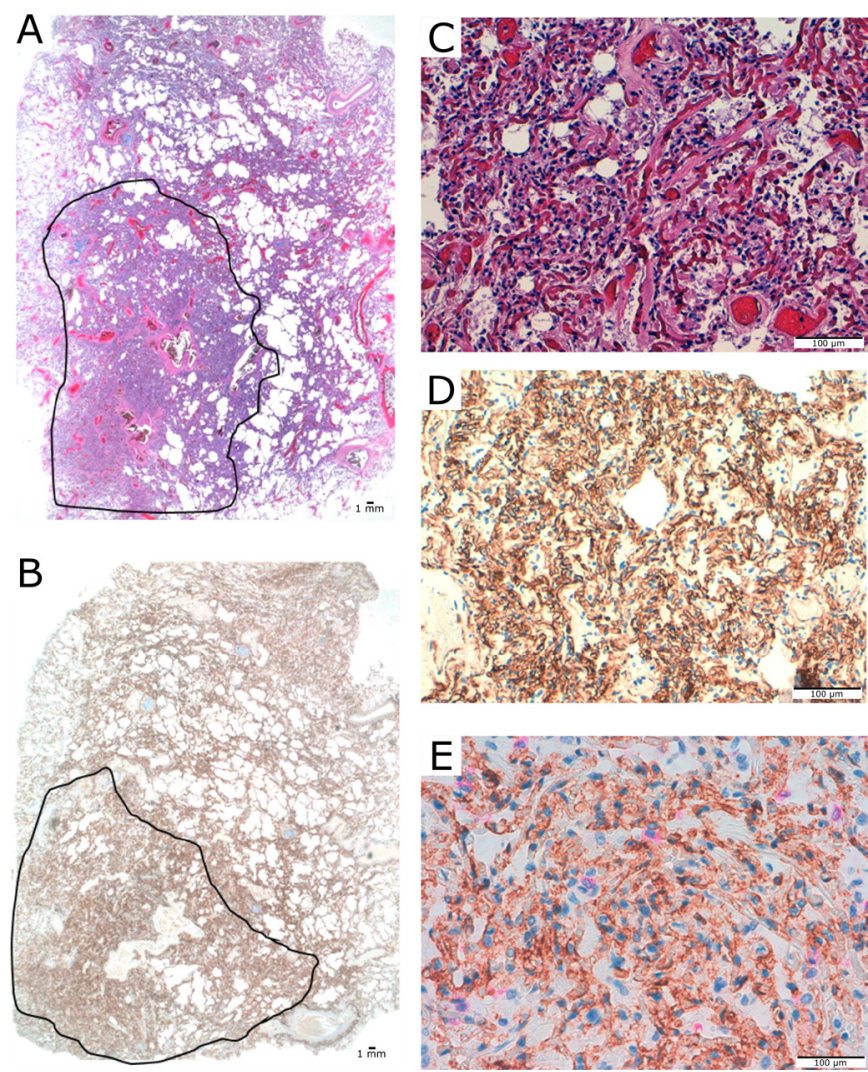

Figure 2 Case with vascular score 3. (A) Complex mesh-like vascular framework of $1.7 \mathrm{~cm}$ of diameter with no or minimal residual alveolar spaces (area within lines), panoramic view. (B) CD34 staining, panoramic view. (C) Mesh-like framework capillaries replacing normal lung. Detail of area within lines is shown (A). (D) CD34 staining. (E) Dual staining for CD34/CK18 with scarce epithelial cells in residual small-size alveolar spaces.

However, in our series, we did not observe differences in weight between patients with different VS.

Vascular proliferation likely reduces the ventilation:perfusion ratio, contributing to refractory severe hypoxaemia. However, although we observed that patients with high VS had a lower $\mathrm{PaO}_{2} / \mathrm{FiO}_{2}$ (last measurement before death) than those with low VS $(83.67 \pm 17.14$ vs $94.86 \pm 34.40)$, these differences were not statistically significant, probably due to the small sample size and the confounding effect of DAD lesions present in most patients. We observed that VS increased with age. Increasing age is a
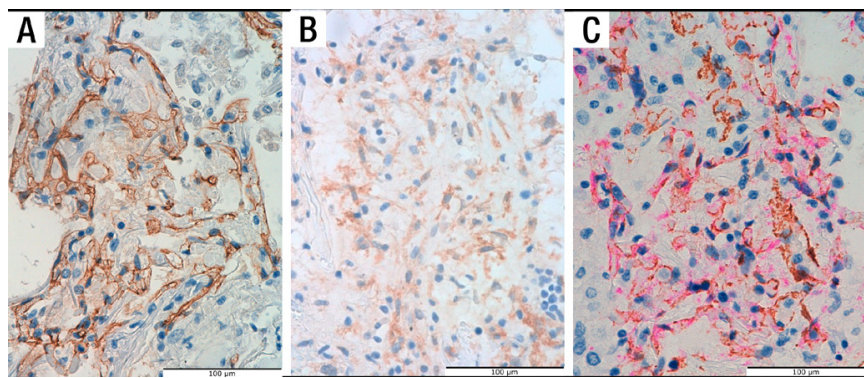

Figure 3 Vascular markers. Positive vascular endothelial cadherin staining in a glomeruloid-like structure (A). Single immunostaining with platelet-derived growth factor receptor- $\beta$ (B) and dual staining for CD34 (pink) and smooth muscle actin (brown) (C), demonstrating the presence of pericytes. 
well-known risk factor for death in patients with COVID-19. Further studies, including a larger number of patients, are necessary to evaluate the possible role of vascular proliferation in the poor prognosis of older patients.

Although poorly characterised at the histological level, increased angiogenesis has been demonstrated after DAD induced by SARS-CoV-2 and influenza A. ${ }^{49}$ After injury, angiogenesis occurs to restore lung vasculature. Ackermann et al demonstrated that the amount of new vessel growth in the lung of patients with COVID-19 was 2.7 times higher than in the lungs from patients with influenza. The authors speculated that the greater degree of endothelialitis and thrombosis (two types of lesions that we also that were observed in our series) in the lungs from patients with COVID-19 may contribute to the increased angiogenesis observed in these patients in comparison with influenza patients. ${ }^{4}$

Although SARS-CoV-2 can infect endothelial cells, we did not observe viral protein expression in our immunohistochemical study (not shown). In vitro studies have reported that alveolar epithelial cells were more susceptible to SARS-CoV-2 infection than pulmonary microvascular endothelial cells. ${ }^{10}$ More probably, endothelial cells can be damaged by excessive exposure to proinflammatory cytokines secreted by epithelial cells, leukocytes and macrophages in the context of DAD. In our series, macrophages were the most frequent inflammatory infiltrate observed, which are well-known producers of proangiogenic factors.

Our retrospective study has several limitations, such as the small sample size and the lack of a validation cohort including patients without COVID-19. Although we do not claim that vascular proliferation is a specific finding in patients with COVID-19, our results support the previous suggestion of increased angiogenesis in this disease. In addition, vascular proliferation provides a plausible histological explanation to some previous clinical and ultrastructural findings in patients with severe COVID-19 and to the pathophysiology of their acute respiratory failure.

Twitter Irene Carretero-Barrio @irenecarretero, Amparo Benito @AmparoBenito and Jose Palacios@jpalacioscalvo

Acknowledgements The authors thank Javier Zamora, from the biostatistics unit of Hospital Universitario Ramón y Cajal (Madrid, Spain), for reading and critically reviewing the manuscript; Marta Rosas and María Luisa Zamorano, from the pathology department of Hospital Universitario Ramón y Cajal (Madrid, Spain), for technical assistance in the histopathological and immunohistochemistry studies; and Hospital Universitario Ramón y Cajal-IRYCIS Biobank (Madrid, Spain), for the management of tissue samples.
Contributors BP-M, MG-R, AC, RdP, JCG, DP and JP contributed to the conception of the study. BP-M, MG-R, IC-B, TB, AB, MG-C, AC and JP contributed to the data collection. BP-M, IC-B, RdP, JCG, DP and JP contributed to data analysis and interpretation. BP-M, IC-B and JP drafted the manuscript. All authors revised the manuscript for intellectual content and approved the final version of the manuscript.

Funding This work was supported by Instituto de Salud Carlos III (grant PI 19/01331), CIBERONC (grant CB16/12/00316) and Instituto Ramón y Cajal de Investigación Sanitaria Intramural COVID19 (project grant 2020/0290).

Competing interests None declared.

Patient consent for publication Not required.

Provenance and peer review Not commissioned; externally peer reviewed.

Open access This is an open access article distributed in accordance with the Creative Commons Attribution Non Commercial (CC BY-NC 4.0) license, which permits others to distribute, remix, adapt, build upon this work non-commercially, and license their derivative works on different terms, provided the original work is properly cited, appropriate credit is given, any changes made indicated, and the use is non-commercial. See: http://creativecommons.org/licenses/by-nc/4.0/.

\section{ORCID iDs}

Irene Carretero-Barrio http://orcid.org/0000-0003-1986-2965

Álvaro Caballero http://orcid.org/0000-0003-1859-0662

Jose Palacios http://orcid.org/0000-0002-6730-5066

\section{REFERENCES}

1 Patel BV, Arachchillage DJ, Ridge CA, et al. Pulmonary angiopathy in severe COVID-19: physiologic, imaging, and hematologic observations. Am J Respir Crit Care Med 2020;202:690-9

2 Si-Mohamed S, Chebib N, Sigovan M, et al. In vivo demonstration of pulmonary microvascular involvement in COVID-19 using dual-energy computed tomography. Eur Respir J 2020;56. doi:10.1183/13993003.02608-2020. [Epub ahead of print: 29 Oct 2020].

3 Thillai M, Patvardhan C, Swietlik EM, et al. Functional respiratory imaging identifies redistribution of pulmonary blood flow in patients with COVID-19. Thorax 2021;76:182-4.

4 Ackermann M, Verleden SE, Kuehnel M, et al. Pulmonary vascular Endothelialitis, thrombosis, and angiogenesis in Covid-19. N Engl J Med 2020;383:120-8.

5 Menter T, Haslbauer JD, Nienhold R, et al. Postmortem examination of COVID-19 patients reveals diffuse alveolar damage with severe capillary congestion and variegated findings in lungs and other organs suggesting vascular dysfunction. Histopathology 2020;77:198-209.

6 De Michele S, Sun Y, Yilmaz MM, et al. Forty postmortem examinations in COVID-19 patients. Am J Clin Pathol 2020;154:748-60.

7 O'Keefe MC, Post MD. Pulmonary capillary hemangiomatosis: a rare cause of pulmonary hypertension. Arch Pathol Lab Med 2015;139:274-7.

8 Haque AK, Gadre S, Taylor J, et al. Pulmonary and cardiovascular complications of obesity: an autopsy study of 76 obese subjects. Arch Pathol Lab Med 2008;132:1397-404.

9 Zhao G, Weiner Al, Neupauer KM, et al. Regeneration of the pulmonary vascular endothelium after viral pneumonia requires COUP-TF2. Sci Adv 2020;6:eabc4493.

10 Wang $P$, Luo $R$, Zhang $M$, et al. A cross-talk between epithelium and endothelium mediates human alveolar-capillary injury during SARS-CoV-2 infection. Cell Death Dis 2020;11:1-17. 